\title{
PROKLA-Redaktion
}

\section{Editorial: Die kommende Deflationskrise?}

Seit den 1960er Jahren war Inflation und ihre Bekämpfung eines der wichtigsten Themen der Wirtschaftspolitik in den entwickelten kapitalistischen Ländern. In der Tat baute sich Ende der 1960er Jahre eine inflationäre Welle auf, die dann Ende der 1970er Jahre und Anfang der 1980er Jahre mit zweistelligen Inflationsraten in vielen industriell entwickelten Ländern ihren Höhepunkt erreichte. In den 1980er Jahren ebbte die Inflationswelle in den Industrieländern ab. Allerdings hatten in dieser Periode viele Entwicklungsländer und später auch nahezu alle Nachfolgestaaten der ehemaligen Sowjetunion mit zum Teil sehr hohen Inflationsraten zu kämpfen, in vielen dieser Länder kam es zu Hyperinflationen mit einem jährlichen Anstieg des Preisniveaus von mehreren Hundert oder gar Tausend Prozent. Allerdings sanken auch hier die Inflationsraten ab Mitte der 1990er Jahre auf durchschnittlich einstellige Raten.

Inflation scheint für die Weltwirtschaft in absehbarer Zeit kein ernsthaftes Problem zu sein. Ganz im Gegenteil, es zeichnet sich eine Entwicklung $a b$, die vor 10 oder 15 Jahren nicht für möglich gehalten wurde: Erneut scheint eine Deflation möglich zu sein, die schon in den 1930er Jahren verheerende Wirkungen für die Weltwirtschaft hatte. Von den industriell entwickelten Ländern musste vor allem Japan ab Mitte der 1990er Jahre eine moderate, jedoch langanhaltende Deflationsphase hinnehmen, die noch nicht überwunden ist. Bei den Entwicklungsländern ist es vor allem die Volksrepublik China, die ab Ende der 1990er
Jahre durch deflationäre Entwicklungen gekennzeichnet ist. Vor dem Hintergrund des konjunkturellen Einbruchs in allen Ökonomien der westlichen Welt sowie der schlechten Lage auch in den meisten Entwicklungsländern setzte der Internationale Währungsfonds eine Task Force ein, welche die Deflationsgefahren in der Weltwirtschaft untersuchen sollte. Im Bericht dieser Task Force vom Frühjahr 2003 zählte Deutschland zusammen mit Japan, Taiwan und Hongkong zur Gruppe der Länder, die als hoch deflationsgefährdet angesehen wurden. Auch die US-amerikanische Zentralbank sah Deflationsgefahren für die USA und die Weltwirtschaft und hat mit schnellen und starken Senkungen ihrer Zinssätze versucht, solchen Tendenzen entgegenzuwirken. Selbst die Europäische Zentralbank sah sich im Mai 2003 dazu gezwungen, ihr Inflationsziel, das sie beim Beginn der Europäischen Währungsunion Anfang 1999 auf zwischen null und zwei Prozent festgelegt hatte, zu präzisieren. Die Zielinflationsrate im Euroraum soll nach der EZB nun leicht unter zwei Prozent liegen. In Deutschland liegt die offiziell gemessene Inflationstate derzeit bei etwa einem Prozent. Berücksichtigt man den breit akzeptierten Sachverhalt, dass durch Qualitätsverbesserungen und neue Produkte die Inflationsrate systematisch zwischen einem und zwei Prozent zu hoch gemessen wird, dann befindet sich Deutschland bereits in einem leichten Deflationsprozess.

$\mathrm{Ob}$ die Weltwirtschaft in den nächsten Jahre von einer Deflationswelle erfasst wird hängt in erster Linie von der Ent- 
wicklung in den USA sowie des DollarKurses ab. Sollıen die USA die nächsten Jahre wie in den 1990er Jahren einen starken und langen Aufschwung erleben und sollte sich der US-Dollar auf seinem jetzigen Niveau stabilisieren, dann wären die Deflationsgefahren in der Weltwirtschaft deutlich reduziert. Sollte der Aufschwung in den USA jedoch schwach bleiben, dann könnten sie nicht emeut als weltweite Konjunkturlokomotive agieren, die mit gigantischen Leistungsbilanzdefiziten die Nachfrage in der gesamten Welt anregt. Selbst ein starkes Wachstum in den USA stabilisiert die Weltwirtschaft nicht automatisch. Denn sollten die internationalen Kapitalströme in die USA, die zur Finanzierung des Leistungsbilanzdefizits notwendig sind, versiegen und der Dollarkurs weiter abstürzen, dann würde das US-amerikanische Leistungsbilanzdefizit schrumpfen. Für die Akkumulationsaussichten der Weltwirtschaft wäre es äußerst problematisch, wenn sich in den USA das Defizit der Leistungsbilanz in einen Überschuss verwandeln sollte. Denn dann würde die Aufwertung des Euro sowic des Yen im Euroraum und in Japan die Deflationsgefahren massiv erhöhen. Besonders verheerend wäre es, wenn der Dollarkurs unkontrolliert ins Trudeln käme und sich die amerikanische Zentralbank gezwungen sähe, durch Hochzinspolitik den Dollarkurs zu verteidigen. In eine solche Konstellation waren die USA schon einmal Ende der 1970er Jahre geraten. Die damals verfolgte harte restriktive Geldpolitik hat nicht nur zu dem bislang tiefsten Konjunktureinbruch in den USA seit den Zweiten Weltkrieg geführt, sondern auch zu einer tiefen Krise der Weltwirtschaft beigetragen, die mit der Zahlungsunfähigkeit Mexikos im Jahre 1982 deutlich sichtbar wurde. Heute hätte eine solche Entwicklung eine noch weitaus fatalere Wirkung als damals, da die Weltwirtschaft heute insgesamt krisenanfälliger geworden ist.

Selbst wenn der sich andeutende weltwirtschaftliche Aufschwung, dessen Stär- ke allerdings noch nicht abzuschätzen ist, die Deflationsgefahren kurzfristig in den Hintergrund drängt, ist in den 1990er Jahren doch eine weltwirtschaftliche Konstellation entstanden, welche überzyklisch deflationäre Gefahren bestehen lässt. Die neue und in der Tendenz deflationäre Konstellation ist mit zwei längerfristigen Entwicklungen verbunden.

Erstens hat die schrittweise vorangetriebene Deregulierung der internationalen Finanzmärkte die Konkurrenz zwischen Währungen intensiviert, da private Haushalte, Unternehmen, Banken etc. die Möglichkeit bekamen, bei ihren Vermögensanlagen effektiv zwischen verschiedenen Währungen zu wählen. Für ihre Anlageentscheidung spielt einerseits die mögliche Kapitalverwertung (Höhe der Zinssätze, erwartete Dividenden, Kurssteigerung der Aktien) andererseits die Sicherheit der Währung eine Rolle und die bemisst sich vor allem an der Abwesenheit von inflationären Prozessen aber auch an der politischen und ökonomischen Stärke des Landes, das die entsprechende Währung ausgibt. In dieser Währungskonkurrenz kann nur bestehen, wer eine "wertstabile" Währung bieten kann. Damit verstärkt die Währungskonkurrenz die ohnehin schon in der Weltwirtschaft bestehende Hierarchie: insbesondere die Länder der sog. „Dritten Welt" können mit den Währungen an der Spitze der Währungshierarchie wie dem US-Dollar oder dem Euro nicht konkurrieren. Die Folgen sind cin hoher "Dollarisierungsgrad" in diesen Ländern, d.h. auch im Inland wird ein großer Teil der Geldfunktionen von einer ausländischen "harten" Währung wie dem Dollar oder dem Euro übernommen, Kapitalexporte in „sichere“ Währungshäfen und der Zwang, internationale Kredite in fremder Währung aufzunehmen, so dass der sowieso schon schwache Schuldner das gesamte Wechselkursrisiko tragen muss, was bei den USA oder den Ländern der Eurozone, die sich in eigener Währung verschulden können, nicht der Fall ist. 
Zweitens hat sich aber auch die Währungskonkurrenz zwischen den führenden Währungen intensiviert. Der USDollar übernimmt nicht mehr so unangefochten wie noch in den letzten Jahrzehnten alle internationalen Geldfunktionen. Mit dem Euro ist zum ersten Mal seit dem Ende des 2. Weltkrieges eine Währung entstanden, welche die Rolle des Dollars als Weltgeld zukünftig ernsthaft in Frage stellen könnte. Die weltpolitischen Konsequenzen einer verschärften wirtschaftlichen Konkurrenz zwischen Europa und den USA wurden bereits in der letzten Ausgabe der PROKLA (Nr. 133, Imperialistische Globalisierung, Dezember 2003) angesprochen. Die ökonomischen Folgen der zunehmenden Währungskonkurrenz sind zunächst einmal heftige Wechsellkursschwankungen zwischen den Währungen an der Spitze der Währungshierarchie und einer Tendenz zu einer restriktiven, vor allem die "Geldwertstabilität" sichernden Geldpolitik, denn auch die führenden Währungen der Welt müssen sich vor den internationalen Anlegern bewähren, wenn sie nicht durch Kapitalverlagerungen „bestraft" werden wollen.

Insgesamt hat die Deregulierungswelle und die verstärkte Währungskonkurrenz zu einem strukturellen Machtgewinn von Geldvermögensbesitzern geführt. Der weltweite Rückgang von Inflationsraten, die zunehmende Unabhängigkeit von Zentralbanken, die Verabsolutierung der Dominanz von Preisniveaustabilität gegenüber allen anderen Zielen der Wirtschaftspolitik, die in vielen Ländern zu beobachten ist, sind Ausdruck dieser Machtverschiebung. Eine Konsequenz dieser neuen Situation, die bislang noch kaum diskutiert wurde, ist die latent deflationäre Konstellation der Weltwirtschaft. Hansjörg Herr gibt in seinem Beitrag einen Überblick über die verschiedenen Dimensionen der Deflation und verdeutlicht, wie die Weltwirtschaft im Zuge der Globalisierung vor allem der Finanzmärkte in diese neue Konstellation geraten ist.

\section{Warum sind Deflationen so gefährlich?}

Fallende Preise sind für die Haushalte zwar zunächst angenehm, da sie deren reale Kaufkraft erhöhen. Für die Dynamik einer kapitalistischen Ölkonomie, deren Akkumulation zu einem großen Teil kreditfinanziert verläuft, ist eine Deflation allerdings fatal, denn sie erhöht ganz allgemein die reale Belastung der Schuldner: Unternehmen realisieren deflationsbedingt geringere Umsatzerlöse, die Rückzahlung ihrer Schulden ist aber nominell fixiert ist, so dass es schwieriger für sie wird, die Schulden zu bedienen. Dasselbe gilt im übrigen auch für den Staat, der aufgrund der Deflation geringere Steuereinnahmen realisiert. Deflationen können $a b$ einer gewissen Intensität zum Zusammenbruch von besonders stark verschuldeten Unternehmen wie auch zu verschärften Finanzproblemen der öffentlichen Haushalte führen. Die Zahlungsprobleme der Schuldner führen dann typischerweise zu einer Bankenkrise, die eventuell den letzten Damm gegen eine große Depression einreißt. Die Weltwirtschaftskrise der 1930er Jahre kann als Anschauungsmaterial für diesen Fall gelten.

Deflationen können auch zum Zusammenbruch der Nachfrage auf den Gïtermärkten führen. Ein Unternehmen wird heute nicht investieren, wenn es damit rechnen muss, dass ein Konkurrent die gleiche Maschine oder das gleiche Gebäude zu einem späteren Zeitpunkt günstiger kaufen kann. Ebenso werden Konsumenten bei Deflationserwartungen die Käufe von langlebigen Konsumgütern und Immobilien in die Zukunft verschieben, so dass auch diese Nachfragekomponente bei eine Deflation massiv geschwächt wird, was die Investitionszurückhaltung der Unternehmen noch verstärkt.

Die Geldpolitik der Zentralbank, die bei inflationären Prozessen mit einer Erhöhung der Leitzinsen gegensteuern kann, wird bei Deflationen weitgehend wir- 
kungslos. Eine Zentralbank kann zwar ihren Refinanzierungszinssatz aúf Nüll senken, wie in Japan geschehen, aber nicht unter Null. Daher steigt mit der Deflationsrate die Belastung der Schuldner, ohne dass die Zentralbank mit ihrer Geldpolitik etwas dagegen unternehmen könnte. Einer Deflation kann der Staat dann nur noch durch Fiskalpolitik, d.h. durch Steuersenkungen oder Ausgabenerhöhungen begegnen. In Japan hat die Fiskalpolitik bisher dazu beigetragen, dass die Deflation sich nicht kumulativ entwickelt hat. Auch in den USA schaltete die Fiskalpolitik schnell auf einen expansiven Kurs um, allerdings wurden dort durch die massiven Steuererleichterungen vor allem die Bezieher hoher Einkommen und die Unternehmen begünstigt. Europa leidet dagegen am Stabilitäts- und Wachstumspakt, der ein maximales Budgetdefizit von nicht mehr als drei Prozent des Bruttoinlandsprodukt festlegt. Zwar wurde der Pakt vor allem von Frankreich und Deutschland gebrochen, da der konjunkturell bedingte Einbruch der Steuern schlicht und einfach nicht durch Ausgabenkürzungen zu kompensieren war, jedoch wird zumindest in Deutschland nach wie vor eine Sparpolitik betrieben, die die deflationären und krisenhaften Tendenzen eher verstärkt. Sollte es in den nächsten beiden Jahren in Deutschland tatsächlich zu einem leichten konjunkturellen Aufschwung kommen, dann jedenfalls nicht wegen, sondern trotz der Wirtschaftsund Finanzpolitik der rot-grünen Regierung.

\section{Wo kommen Deflationen her?}

Letztlich finden Preisniveauänderungen ihre Ursachen in sich verändernden Kosten. Steigt das Kostenniveau in einer Ökonomie, so führt dies zu Inflation, da die Unternehmen, um Verluste zu vermeiden, gestiegene Kosten auf die Preise überwälzen. Da bei allgemeinen Kostenerhöhungen alle Unternehmen betroffen sind, ist die Überwälzung auf die Preise einfach und wird nicht durch die Konkurrenz zwischen Unternehmen verhindert. Bei Deflationen findet der umgekehrte Prozess statt. Allgemeine Kostensenkungen führen zu allgemeinen Preissenkungen.

Eine der wichtigsten Kostenkomponenten sind die Lohnstückkosten. Sinken diese, dann kommt es zu einem sinkenden Preisniveau. Die Lohnstïckkosten hängen von der Veränderung der Stundenlöhne und der Arbeitsproduktivität $a b$. Sobald die Lohnerhöhungen unter dem Anstieg der Produktivität liegen oder es gar zu Lohnsenkungen kommt, sinken Lohnstückkosten und Preisniveau. In diesem Fall reißt der "Lohnanker", der eine Deflation verhindern kann. Empirisch ist der Zusammenhang zwischen der Entwicklung der Lohnstückkosten und dem Preisniveaus ausgesprochen eng. Cornelia Kaiser zeigt in ihrem Beitrag, dass die zentrale Ursache der Deflation in Japan das Reißen des Lohnankers nach dem Platzen der Börsenblase Ende der 1980er Jahre war. Sie macht deutlich, dass die unternehmensbezogenen Lohnverhandlungen in Japan, die im Falle yon ökonomischen Problemen im Unternehmen, bei den Beschäftigten dazu führen, dass sic es in Kauf nehmen, dass ihre Löhne nur schwach oder auch gar nicht steigen, den Kern des Deflationsprozesse ausmachen: Wenn alle Unternehmen versuchen, sich durch niedrige Lohnerhöhungen im Konkurrenzprozess einen Vorteil zu verschaffen, dann resultiert eine Deflation, in der alle verlieren. Eine weitere Kostenkomponente sind Wechselkursveränderungen. Eine Aufwertung der eigenen Währung verbilligt importierte Güter und wirkt dadurch deflationär. Die Gefahr weiterer Aufwertungen des Yen und des Euro ist dann auch einer der Gründe, die den Internationalen Währungsfonds zur Überzeugung kommen ließ, dass Japan und Deutschland hochgradig deflationsgefährdet sind. Veränderungen der Steuern und Sozialabgaben oder Veränderungen von Rohstoffpreisen wirken ebenfalls über die 
Kostenseite auf das Preisniveau ein.

Auch die Nachfrage aul den Gütermärkten kann inflationär oder eben auch deflationär wirken. Leidet der Unternehmenssektor an einem Nachfragemangel, dann nimmt der Druck in Richtung Preissenkungen zu. Oben wurde betont, dass eine Deflation von sich aus die Investitions- und Konsumnachfrage absenkt. Typisch für einen Deflationsprozess sind somit sinkende Kosten und ein Nachfragemangel. Kosten- und Nachfragedeflation verstärken sich in diesem Fall und führen zu den ökonomischen Zerrüttungstendenzen, die aus deflationären Phasen in der Geschichte bekannt sind.

Ein ähnlicher Effekt wie bei einer Deflation ergibt sich, wenn ein Land, das in fremder Währung verschuldet ist, seine eigene Währung abwerten muss. Denn auch in diesem Fall bleibt der in fremder Währung definierte Schuldendienst unverändert, während die Einnahmen eines inländischen Unternehmens, selbst bei gleicher Umsatzsumme in inländischer Währung, unter Umständen nicht mehr ausreichen, um den Schuldendienst zu leisten, da sich der selbe Betrag inländischer Währung jetzt gegen einen geringeren Betrag der ausländischen Währung umtauscht. Aufgrund dieses Mechanismus führen Währungskrisen bei Schulden in Fremdwährung immer auch zu Unternehmenszusammenbrüchen und Bankenkrisen im abwertenden Land. Die Belastung von Schuldnern steigt somit bei einem Fall des Preisniveaus ebenso wie bei einer Abwertung und Verschuldung in Fremdwährung. Elmar Altvater arbeitet in seinem Beitrag genau diesen Aspekt des Deflationsprozesses, die Verteuerung des Kapitals für die Schuldner und das Ansteigen der Realzinsen mit all seinen fatalen Konsequenzen heraus.

\section{Die groteske wirtschaftspolitische Debatte in Deutschland}

In Deutschland ging die Debatte um Deflationsgefahren an der offiziellen Wirtschaftspolitik weitgehend vorbei. Die Regierung hat über Jahre versucht, mit allen Mitteln den Stabilitäts- und Wachstumspakt zu erfülien. Sie ist in erster Linie aufgrund der einbrechenden Steuereinnahmen dann aber doch gescheitert. Von einer aktiven antizyklischen Politik der Bundesregierung ist nichts zu spüren. Im Gegenteil, die Logik von Sachzwängen wird ins Feld geführt, um Ausgaben radikal zu kürzen. Die öffentlichen Investitionen sind seit Jahren im Keller, da die Gemeinden, die sich nur sehr begrenzt verschulden können, aufgrund ihrer finanzieller Engpässe in erster Linie an den investiven Ausgaben sparen. Der Sozialstaat wird an allen Ecken und Enden gestutzt, um die Ausgaben der öffentlichen Haushalte einschließlich der Haushalte der Sozialversicherungen den sinkenden Einnahmen anzupassen. In einigen Bundesländern - der Vorreiter war der rot-rote Senat in Berlin - hat man mit der Zerstörung des Flächentarifvertrages und deutlichen Lohnkürzungen der im öffentlichen Bereich Beschäftigten begonnen. Zwar ist eine ausufernde Staatsverschuldung auch nicht die Lösung aller Probleme, aber ausgerechnet in einer ökonomischen Krisenphase die Konsolidierung der öffentlichen Haushalte zu betreiben, verstärkt eher eine Abwärtsspirale von sinkenden Einnahmen und noch größeren Haushaltslöchern. Insofern wird man von der gegenwärtigen rot-grünen Haushaltspolitik unangenehm an die Fiskalpolitik der Regierung Brüning erinnert, die mit ähnlichen Schritten die Krise der 1930er Jahre massiv verstärkt hat. Unter anderem waren damals die Lohnsenkungen im öffentlichen Bereich das Signal für allgemeine Lohnkürzungen. Brüning hat wie heute seine restriktive Fiskalpolitik mit Sachzwängen begründet. In der Tat waren seine Sachzwänge aufgrund der da- 
maligen Reparationszahlungen deutlich größer als es heute der Fall ist. Michael Heine untersucht in seinem Beitrag die Fiskalpolitik der Brüning-Zeit und zieht Konsequenzen für die derzeitige Politik der öffentlichen Haushalte in Deutschland.

Deflationen können kumulativen Charakter annehmen, wenn die Geldlöhne nicht entsprechend der Produktivität ansteigen oder gar anfangen $\mathrm{zu}$ sinken. Denn dann wird der nominelle Anker des Preisniveaus zerstört. In Deutschland zeigen sich derzeit verstärkte Tendenzen, dass dieser "Lohnanker" reißt. Verschiedene Effekte spielen hier zusammen: Die Unternehmen bauen freiwillige, übertarifliche Zahlungen $a b$, die Gewerkschaften sind aufgrund anhaltender Massenarbeitslosigkeit und der politischen Gesamtlage geschwächt und nicht in der Lage wirkliche Lohnerhöhungen durchzusetzen, und es gibt eine breite Allianz von Politikern, Ökonomen und Wirtschaftsjournalisten, die dem Glauben anhängen, man könne über allgemeine Lohnsenkungen die Arbeitslosigkeit verringern. Selbst Gewerkschaften haben sich im Rahmen von „Bündnissen für Arbeit" partiell auf diese Logik eingelassen. Zur Kritik solcher Vorstellungen ist nach wie vor gültig, was in PROKLA 116 (,Rot-Grüner“ Absturz, September 1999) bereits ein Jahr nach Antritt der ersten rot-grünen Regierung geschrieben wurde. In ihrem Beitrag zeigen Eckhard Hein, Thorsten Schulten und Achim Truger, dass die gegenwärtige Entwicklung der Arbeitsmärkte in Deutschland die Deflation verstärkt, so dass Deutschland am Rande einer Deflationsspirale steht. Sie machen weiter deutlich, dass aufgrund der Bedeutung Deutschlands für den Euroraum die Gefahr besteht, dass eine deflationäre Entwicklung in Deutschland die EU mitreißt. Denn es ist in der EU bisher nicht gelungen, einen Lohnbildungsmechanismus zu etablieren, der einen Damm gegen deflationäre Entwicklungen sein könnte.
Das Thema Deflation wird also aktuell bleiben, sowolnl auf nationaler und auf europäischer Ebene, wie auch auf der Ebene der Weltwirtschaft.

$$
* * *
$$

Bereits im letzten Heft wurde angekündigt, dass zukünftig kürzeren Debattenbeiträgen zu aktuellen Themen ein gröBerer Raum als bisher eingeräumt werden soll. Für solche Beiträge haben wir jetzt die Rubrik „Einsprüche" geschaffen. Wir eröffnen sie in diesem Heft mit einem Beitrag von Jan Hoffrogge zu den studentischen Streiks im vergangenen Wintersemester sowie einem Kommentar von Stephan Lessenich zu aktuellen sozialpolitischen Debatten und Vorurteilen. Ein früherer Beitrag von Stefanie Hürtgen zu Fragen der Gewerkschaftspolitik (PROKLA 130 Gewerkschaften zur Sonne, zur Freiheit?, März 2003) hat inzwischen Wellen auch in anderen Zeitschriften geschlagen: Richard Detje antwortete in Sozialismus $9 / 03$ auf die Kritik von Hürtgen und auf diese Antikritik reagierte Hubert Herfurth in express 11$12 / 2003$.

$$
* * x
$$

Es bleibt noch, eine traurige Mitteilung zu machen. Hansgeorg Conert ist im Januar in Bremen verstorben. Er war (inzwischen emeritierter) Hochschullehrer an der Universität Bremen und über lange Jahre Mitglied in der Vereinigung zur Kritik der politischen Ökonomie, von det die PROKLA herausgegeben wird. In der PROKLA analysierte er schon früh die Widersprüche der „realsozialistischen" Gesellschaften. Volker Stork schrieb einen Nachruf über den Linkssozialisten und Individualisten Hansgeorg Conert. 